\title{
DESIGN OF HELMHOLTZ RESONATOR WITH REQUIRED CHARACTERISTICS
}

\author{
Aleksei B ykov * and Alexandr Komkin \\ B M STU, Power Engineering Department, 105005, M oscow, Russia
}

\begin{abstract}
The method of calculation design of the Helmholtz resonator in the duct is considered based on the transition to dimensionless parameters, which are determined concerning the transverse size of the duct. The acoustical impedance of $\mathrm{Helmholtz}$ resonator is analyzed. The application of this approach to the special case of a short-neck resonator and the general case with a linear operating mode of the resonator is described.
\end{abstract}

\section{Introduction}

Helmholtz resonator refers to one of the main and most common elements in noise reduction systems for various machines and equipment [1-4]. A fairly large number of publications [522] are devoted to the Helmholtz resonator calculation, in which various aspects of this problem are considered. They mainly include an estimate of the natural frequency of the resonator and analysis of the influence of the geometric configuration of the resonator on its acoustic characteristics.

The acoustic efficiency of Helmholtz resonator can be increased by the correct selection of its geometric parameters. A detailed discussion of this issue concerning an isolated resonator and a resonator in an infinite wall was carried out by Ingard in his fundamental work [5]. From a practical point of view, it is advisable to extend the approach of Ingard for the case of the Helmholtz resonator placed on the duct wall. For such a configuration (fig. 1), to three parameters that characterize the resonator itself, one more parameter is added that determines the transverse dimensions of the duct. The consideration of this problem was begun in $[16,17]$. In [18], a solution was obtained for the case when the resonator neck length can be neglected. This work is a continuation of studies in this direction to extend the results to the more general case.

\section{Acoustic characteristics of Helmholtz resonator}

Let us consider a direct duct (Figure 1) with absolutely rigid walls and with a cross-sectional area $S$, on the duct wall of which a Helmholtz resonator is placed. We will consider the simplest Helmholtz resonator model, in which a rigid closed resonator surface, characterized only by volume $\mathrm{V}$, is attached to the duct through the neck with a cross-sectional area $S_{0}$ and a length $l$. It is assumed that the dimensions of the resonator are much smaller than the

* Corresponding author: alecksbykov@ mail.ru 
considered wavelengths. We assume that the duct is narrow so that only plane sound waves can propagate in it.

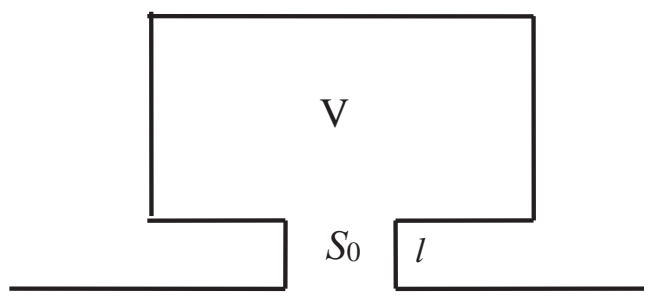

$S$

Fig. 1. Helmholtz resonator on the duct wall.

Let us consider a direct duct (Figure 1) with absolutely rigid walls and with a crosssectional area $S$, on the duct wall of which a Helmholtz resonator is placed. We will consider the simplest Helmholtz resonator model, in which a rigid closed resonator surface, characterized only by volume $\mathrm{V}$, is attached to the duct through the neck with a crosssectional area $S_{0}$ and a length $l$. It is assumed that the dimensions of the resonator are much smaller than the considered wavelengths. We assume that the duct is narrow so that only plane sound waves can propagate in it.

The natural frequency of the Helmholtz resonator $f_{0}$ is defined as [16]:

$$
f_{0}=\frac{c}{2 \pi} \sqrt{\frac{S_{0}}{l_{e} \mathrm{~V}}}
$$

where c is the speed of sound; $\mathrm{V}$ is the volume of the resonator, $S_{0}$ is the cross-sectional area of the neck of the resonator (for a circular neck with a diameter $d_{0}, S_{0}=\pi d_{0}^{2} / 4$ ); $l_{e}$ is the effective length of the neck of the resonator, $l_{e}=l+l_{a} ; l$ is the actual length of the neck of the resonator; la is the attached length, determined by the redistribution of sound energy to the fading higher oscillation modes on both sides of the resonator's neck; $l_{a}=l_{a \mathrm{~V}}+l_{a s} ; l_{a \mathrm{~V}}$ is the internal attached length of the neck (from the cavity side); $l_{a s}$ is the external attached neck length (from the duct side).

The attached neck length la substantially depends on the geometric configuration of the resonator-duct system. For definiteness, let us assume that the characteristic transverse size of the neck of the resonator (for a circular neck its diameter) is $d_{0}$ and the cavity of the resonator is formed by a cube with side $L$ so that its volume is $\mathrm{V}=L^{3}$. If we introduce into consideration the dimensionless parameter $g_{\mathrm{V}}=d_{0} / L$, then the attached length $l_{a \mathrm{~V}}$ for not too large $g_{\mathrm{V}}$ values (less than 0.5 ) can be approximated with sufficient precision for practice by the formula [19]:

$$
l_{a V}=\left(0.815-1.09 g_{V}\right) d_{0} / 2
$$

For definiteness, we also assume that the duct has a square section with side $b$ so that its area is $S=b^{2}$. Then, if we introduce one more dimensionless parameter $g=d_{0} / b$, the attached length $l_{a s}$ for not too large $g$ values (less than 0.5 ) can be accurately described with sufficient precision for practice by the approximating dependence [16]: 


$$
l_{a S}=(0.815-0.625 g) d_{0} / 2
$$

Then the total attached neck length $l_{a}$ can be written in the form:

$$
l_{a}=\left(0.815-0.546 g_{V}-0.313 g\right) d_{0}=\left(\alpha-\beta_{V} g_{V}-\beta_{S} g\right) d_{0}
$$

If we introduce a notation $\bar{L}=L / b$, then the last expression can be represented as

$$
l_{a}=\left[\alpha-\left(\beta_{S}+\beta_{V} / \bar{L}\right) g\right] d_{0}
$$

Another important acoustic characteristic of the Helmholtz resonator is the transmission loss [16]:

$$
T L=10 \lg \left[1+\frac{1+2 R_{s}}{R_{s}^{2}+[Q(\Omega-1 / \Omega)]^{2}}\right]
$$

where $R_{s}$ is the reduced resonator resistance, $R_{s}=2 m \bar{R}_{0} ; m=S / S_{0} ; \bar{R}_{0}=R_{0} /(\rho c)$ is the dimensionless resistance of the resonator [16], $\Omega$ is the relative frequency, $\Omega=f / f_{0} ; Q$ is the quality factor of the system [16]

$$
Q=2 m \sqrt{\frac{S_{0} l_{e}}{\mathrm{~V}}}
$$

As follows from (6), at the natural frequency, when $\Omega=1$, the transmission loss depends only on the reduced resistance and reaches its maximum value:

$$
T L_{0}=10 \lg \left[1+\left(1+2 R_{s}\right) / R_{s}^{2}\right]=20 \lg \left[1+1 / R_{s}\right]
$$

The bandwidth of the Helmholtz resonator is determined from the condition that at the boundary frequencies of this band $\Omega_{1,2}$ the transmission loss $T L=3 \mathrm{~dB}$. Moreover, the expression itself under the logarithm in (6) will be equal to 2 . This leads to the following equation for determining the boundary frequencies:

$$
Q\left(\Omega_{1,2}-1 / \Omega_{1,2}\right)=K
$$

when $K=\sqrt{1+2 R_{s}-R_{s}^{2}}$. Obviously, this equation has a solution when its right-hand side takes positive values, i.e. when $K^{2}=1+2 R_{s}-R_{s}^{2}>0$. This condition imposes a limitation on the value of the reduced resonator resistance, the form is:

$$
R_{s}<1+\sqrt{2} \approx 2.4
$$

The solution of equation (9) gives the following expressions for the boundary frequencies

$$
\Omega_{1,2}=\mp \frac{K}{2 Q}+\sqrt{\frac{K^{2}}{4 Q^{2}}+1}
$$


From here we find the relative bandwidth

$$
\Delta \Omega=\Omega_{2}-\Omega_{1}=K / Q
$$

Obviously, with an increase in the reduced resistance, the parameter $K$ decreases, and hence the bandwidth $\Delta \Omega$ decreases. On the other hand, when the reduced resistance $R_{s} \rightarrow 0$ is the parameter $K \rightarrow 1$. In this case, the relative width of the resonance curve is

$$
\Delta \Omega=\frac{1}{Q}=\frac{1}{2 S} \sqrt{\frac{V S_{0}}{l_{e}}}
$$

Thus, the bandwidth $\Delta \Omega$ is inversely proportional to the quality factor $Q$ of the system and depends on all four of its geometric parameters.

At the same time, if we go from relative bandwidth $\Delta \Omega$ to the absolute bandwidth

$$
\Delta f=f_{0} \Delta \Omega=\frac{c}{4 \pi} \frac{S_{0}}{S l_{e}}
$$

then this quantity, in contrast to $\Delta \Omega$, no longer depends on the cavity volume $\mathrm{V}$.

\section{Dimensionless parameters of Helmholtz resonator}

Let us note that the considered system the Helmholtz resonator on the wall duct is characterized by four geometric parameters: three of the parameters characterize the resonator itself (resonator volume $\mathrm{V}$, neck area $S_{0}$ and its length $l$ ), one more is added to determine the transverse dimensions of the duct (its area $S$ ).

On the other hand, calculations usually use the natural frequency of the resonator $f_{0}$ and the bandwidth $\Delta f$ as initial data, which are determined by the geometric parameters of the system. In addition, for this purpose, the value of the transmission loss of the system in question at the natural frequency of the resonator $T L_{0}$ can also be used. As a result, we can obtain a system of three related equations with four unknowns, which does not allow us to select the resonator parameters uniquely and can lead to solutions that are far from optimal.

To eliminate this problem, we will make the transition to dimensionless values, using for this the transverse dimension of the duct $b$. For this purpose, in addition to the dimensionless parameters $g$ and $g_{\mathrm{V}}$ introduced earlier, the dimensionless natural frequency of the resonator $\mu_{0}=f_{0} b / c$ and the relative bandwidth $\Delta \mu=\Delta f b / c$, the dimensionless volume of the resonator cavity $\overline{\mathrm{V}}=\mathrm{V} / b^{3}=\vec{L}^{3}$, and the dimensionless effective neck length $\bar{l}_{e}=l_{e} / d_{0}=\left(l+l_{a}\right) / d_{0}$ are also introduced.

This approach allows us to rewrite formulas (1) and (14) in the form:

$$
\begin{aligned}
& \mu_{0}=\frac{b}{2 \pi} \sqrt{\frac{S_{0}}{l_{e} \mathrm{~V}}}=\sqrt{\frac{g}{16 \pi \bar{l}_{e} \overline{\mathrm{V}}}} \\
& \Delta \mu=\frac{b S_{0}}{4 \pi S l_{e}}=\frac{g}{16 \bar{l}_{e}}
\end{aligned}
$$


The expressions for the dimensionless frequency (15) and the relative bandwidth (16) are related by the equation:

$$
\Delta \mu=\frac{g}{16 \bar{l}_{e}}=\pi \overline{\mathrm{V}} \mu_{0}^{2}
$$

The equation (17) relates the acoustic parameters of the system with its geometrical parameters so that it can be considered the main equation describing the Helmholtz resonator on the duct wall.

Also, from (17) follows the relation

$$
\frac{\Delta \mu}{\mu_{0}^{2}}=\pi \overline{\mathrm{V}}
$$

according to which the ratio of dimensionless bandwidth to square of natural frequency is proportional to the dimensionless resonator volume.

Expanding the dimensionless effective neck length of the resonator in expressions (15) and (16), taking into account (5) we can obtain these expressions to the form

$$
\begin{aligned}
\mu_{0}^{2} & =\frac{g}{16 \pi\left[\bar{l}+\alpha-\left(\beta_{S}+\beta_{V} / \bar{L}\right) g\right] \bar{L}^{3}} \\
\Delta \mu & =\frac{g}{16\left[\bar{l}+\alpha-\left(\beta_{S}+\beta_{V} / \bar{L}\right) g\right]}
\end{aligned}
$$

It follows from (19) that at first, for small values of the parameter $g_{S}$ (diameter $d_{0}$ ), the square of the natural frequency $\mu_{0}^{2}$ depends linearly on this parameter. With a further increase in $g_{S}$, the neck attached length begins to noticeable decrease, which leads to more intensive growth of $\mu_{0}^{2}$ than in the case of linear dependence. A similar dependence is also observed between the parameter $g_{S}$ and the bandwidth $\Delta \mu$.

\section{Selection of geometric parameters of Helmholtz resonator without losses}

Let us assume that the resonator neck is short so that the relation is satisfied, i.e. when the length of the neck is much smaller than its diameter. In this case, equations (15) and (16) can be transformed to:

$$
\begin{aligned}
& g=\frac{16 \pi \mu_{0}^{2} \alpha \bar{L}^{3}}{1+16 \pi \mu_{0}^{2}\left(\beta_{S} \bar{L}+\beta_{V}\right) \bar{L}^{2}} \\
& g=\frac{16 \Delta \mu \alpha}{1+16 \Delta \mu\left(\beta_{S}+\beta_{V} / \bar{L}\right)}
\end{aligned}
$$

Such a representation of the initial equations allows one to relate to each other the natural frequency of the resonator and the bandwidth $\Delta \mu$, as well as the volume of the cavity of the resonator and the parameters of the attached neck length of the resonator, determined by the coefficients $\alpha$ and $\beta$, through the dimensionless neck diameter $g$. The nomograms constructed on the basis of (21) and (22) and presented in Figure 2 allow us to uniquely determine the geometric parameters of the Helmholtz resonator with a short neck, based on the 
requirements for its acoustic characteristics. So, for example, if you set the natural frequency $\mu_{0}$ of the resonator and the required bandwidth $\Delta \mu$ as the initial data, then this unambiguously determines the dimensionless diameter of the resonator neck $g$, and then the volume of the resonator chamber $\bar{V}$. If we take the natural frequency $\mu_{0}$ of the resonator and its volume $\bar{V}$ as the initial data, then this allows immediately determine the dimensionless diameter $g$, and then its bandwidth $\Delta \mu$.

Fig. 2. Nomogram for determining the geometric parameters of a Helmholtz resonator with a short

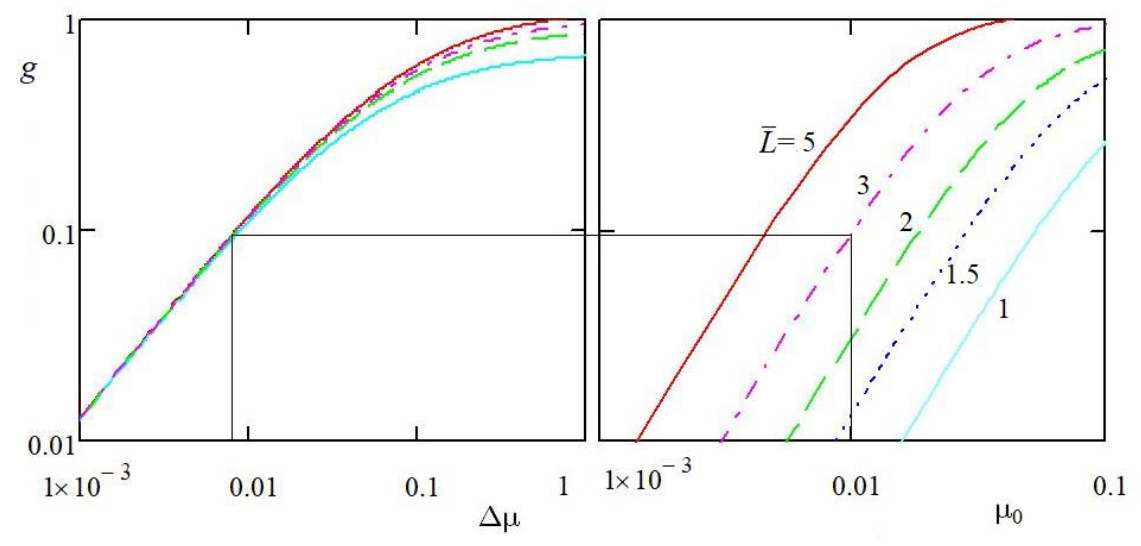

neck.

The graphs in Figure 2 show that to increase the bandwidth the value of the parameter g should be increased, which means essentially an increase in the transverse size of the neck resonator. On the other hand, as follows from another graph, large values of $g$ will require a larger volume of the resonator for a given natural frequency.

We also note that, as follows from the left graph of the presented nomogram, the parameter $\bar{L}=L / b$ begins to influence the dependence of $g$ on $\Delta \mu$ at large values $(g>0.3)$. However, such values of $g$ are usually not realized in practice so that this influence can be neglected.

\section{Acoustic impedance of Helmholtz resonator}

Equation (8) which determines the transmission loss of the system at the natural frequency of the resonator should also be added to equations (19) and (20). Equation (8) for the subsequent consideration can be represented in the form:

$$
T L_{0}=20 \lg \left[1+1 /\left(R_{S}\right)\right]=20 \lg \left[1+g^{2} /\left(2 \bar{R}_{0}\right)\right]
$$

The resonator resistance due to the energy loss of the sound wave during its interaction with the resonator can be divided into two types: linear and non-linear.

Let us first consider the linear resistance of the resonator, which is caused by losses in the viscous and thermal boundary layers on the internal surfaces of the resonator. In this case, viscous losses besides losses at the neck wall and the resonator wall in the vicinity of the neck also include losses at the edges of the neck of the resonator.

The loss of sound energy in the neck of the resonator is determined by the dimensionless viscous resistance, described by the expression [20] 


$$
\bar{R}_{v 0}=2 k \delta_{v} \bar{l}=F_{0} \bar{l}
$$

which under normal environmental conditions is reduced to the form:

$$
\bar{R}_{v 0}=10^{-4} \sqrt{f_{0} l}
$$

For viscous losses estimation on the front wall, cavity of the resonator and on the edges of the neck it is necessary to introduce additional proportionality coefficients $N$ and $E$ [20]. In this case, the total viscous resistance of the resonator can be written in the form:

$$
\bar{R}_{v}=F_{0}(\bar{l}+N+E)
$$

For small neck diameters, when $g \leq 0.1$, as shown in [21], $N=0.28$ can be taken. Estimating the coefficient $E$ is a rather complicated task. Following [20], for a neck of small length in the absence of the influence of the back wall of the cavity of the resonator, the coefficient $E$ can be taken equal to 0.85 . Let us note that the viscous loss of sound energy at the edges of the neck does not depend on the diameter of the neck but can vary significantly with the relative length of this neck. To determine the viscous losses at the edges of the neck, depending on the relative length of the neck of the resonator, it is recommended to use the following approximation of the dependence for the coefficient $E$ :

$$
E=0.8 \bar{l}+0.7
$$

With this in mind, the total viscous losses in the resonator (26) are written in the form:

$$
\bar{R}_{\mathrm{v}} \approx F_{0}(1.8 \bar{l}+1)
$$

As for heat losses, they are determined by dimensionless thermal resistance [20]:

$$
\bar{R}_{\chi}=S_{0} S_{S} \delta_{\chi} /\left(k \mathrm{~V}^{2}\right)
$$

where $S_{S}$ is the area of the internal surface of the cavity of the resonator.

Moreover, from (24) and (29) follows the relationship between thermal and viscous resistances:

$$
\frac{\bar{R}_{\chi}}{\bar{R}_{v 0}}=\frac{\delta_{\chi}}{\delta_{v}} \frac{S_{0} S_{S}}{2 k^{2} \mathrm{~V}^{2} \bar{l}}
$$

which at the natural frequency of the resonator defined by formula (1):

$$
\frac{\bar{R}_{\chi}}{\bar{R}_{v 0}}=\frac{\delta_{\chi}}{\delta_{v}} \frac{S_{S} l_{e}}{2 \mathrm{~V} \bar{l}}
$$

For the considered configuration of the system Helmholtz resonator-duct with $S_{S}=6 L^{2}$ and $\mathrm{V}=L^{3}$. The ratio of the thicknesses of the boundary layers can be considered as $\delta_{\chi} / \delta_{v}=\sqrt{\gamma}$, 
where $\gamma=1,4$ - is the adiabatic exponent, so that formula (5.31) can be transformed to the form

$$
\frac{\bar{R}_{\chi}}{\bar{R}_{v 0}}=3 \sqrt{\gamma} \frac{g_{v}}{\bar{l}}\left(\bar{l}+\bar{l}_{a}\right)
$$

From this, taking into account (24), we obtain the following expression for thermal resistance

$$
\bar{R}_{\chi}=3.5 F_{0} g_{\mathrm{v}}\left(\bar{l}+\bar{l}_{a}\right)
$$

Then the overall dimensionless linear resistance of the resonator is:

$$
\bar{R}_{0 l n}=\bar{R}_{\mathrm{v}}+\bar{R}_{\chi} \approx F_{0}\left[\left(1.8+3.5 g_{\mathrm{v}}\right) \bar{l}+3.5 g_{\mathrm{v}} \bar{l}_{a}+1\right]
$$

For short-neck resonator $(\bar{l} \ll 1)$ it can be presented as:

$$
\bar{R}_{0 l n s}=F_{0}\left[3.5 g_{\mathrm{v}} \bar{l}_{a}+1\right]
$$

Substituting (35) into (23), we obtain the dependence of the transmission loss at the natural frequency of the short-neck resonator on the geometric parameters of the system.

The proposed approach can be extended to the case of the considered system in the developed nonlinearity mode. According to [22], with the results of Chapter 4, the reduced nonlinear resistance of the resonator $\tilde{R}_{0 n l}$ is approximated by the formula

$$
\tilde{R}_{0 n l}=R_{0 n l} /(\rho \sqrt{v \omega})=4.9 \cdot 10^{-3} \tilde{V}_{0}^{2}+140
$$

where $\tilde{V}_{0}$ is the reduced velocity in the neck of the resonator, $\tilde{V}_{0}=V_{0} / \sqrt{v \omega}$, satisfying the condition $\tilde{V}_{0}>100$.

Converting to dimensionless nonlinear resistance in (36), we obtain

$$
\bar{R}_{0 n l}=\frac{R_{0 n l}}{\rho \mathrm{c}}=\frac{\tilde{R}_{0 n l}}{\mathrm{c}} \sqrt{v \omega}=\frac{F_{0}}{2 \sqrt{2}}\left(4.9 \cdot 10^{-3} \tilde{V}_{0}^{2}+140\right)
$$

Such a representation of nonlinear resistance allows its use in formula (23) for estimating transmission losses at the natural frequency of the resonator in the developed nonlinearity modes.

\section{General approach to the selection of Helmholtz resonator parameters}

It should be noted that the calculated transmission loss of the Helmholtz resonator in the duct, if the neck length of the resonator is neglected, can actually be significantly higher. A full account of losses in thermoviscous boundary layers leads to a decrease in the resonator efficiency at the natural frequency. It follows that the introduction of a lower limit on the 
minimum allowable transmission loss at the natural frequency of the resonator $T L_{0 \min }$ is rational. Let us write this condition in the form: $T L_{0} \geq 10 \mathrm{~dB}$. Then from (23) in the first approximation the following equality follows:

$$
g^{2} /\left(2 \bar{R}_{0}\right)=2
$$

From (38) a formula for determining the parameters of the system can be obtained, providing the minimum possible transmission loss. After substituting formula (34) into (38) and subsequent transformations, we obtain:

$$
g=2 \sqrt{F_{0}\left[\left(1.8+3.5 g_{\mathrm{V}}\right) \bar{l}+3.5 g_{\mathrm{v}} \bar{l}_{a}+1\right]}
$$

To this relation, we can add one more relation (20), expressing explicitly the dimensionless diameter of the neck of the resonator:

$$
g=\frac{16 \Delta \mu(\bar{l}+\alpha)}{1+16 \Delta \mu\left(\beta_{S}+\beta_{\mathrm{v}} / \bar{L}\right)}
$$

As show calculations in Figure 2, the second term in parentheses in the denominator in (40) can be neglected, since its influence is insignificant. So that equation (40) can be reduce to the form:

$$
g=\frac{16 \Delta \mu(\bar{l}+\alpha)}{1+16 \Delta \mu \beta_{S}}
$$

Thus, equations (39) and (41) unambiguously relate the transmission loss in Helmholtz resonator $T L_{0}$ at the natural frequency $f_{0}$ to the total resonator resistance $R_{0}$, the dimensionless neck diameter of the resonator $g$, the dimensionless neck length of the resonator $\bar{l}$ and dimensionless parameters of attached length $\alpha$ and $\beta$.

The constructed graphs according to (19) and (20), presented in Figure 3, form a nomogram for determining the parameters of the Helmholtz resonator.

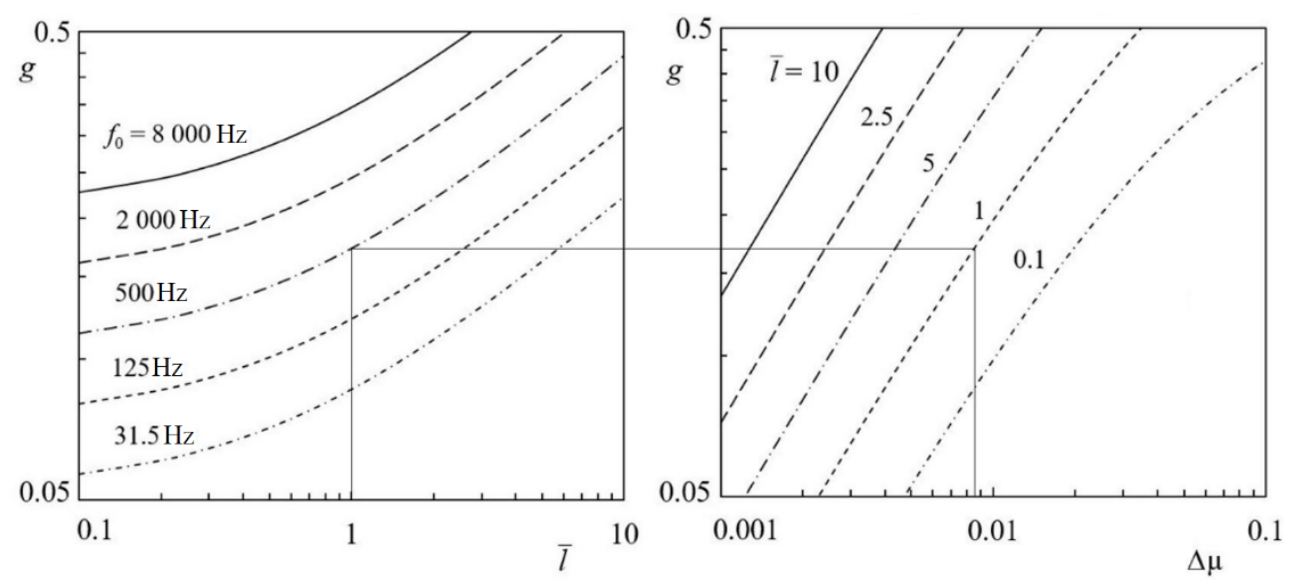

Fig. 3. Nomogram for determining the parameters of the Helmholtz resonator with losses. 
Specifying at this nomogram the natural frequency of the resonator $f_{0}$ and the relative length of the neck of the resonator $\bar{l}$, it is possible to determine the dimensionless relative bandwidth $\Delta \mu$. Further, from (18), the relative volume of the resonator $\bar{V}=\Delta \mu / \mu_{0}{ }^{2}$ can be obtained. Finally, with the given duct diameter $d$, we can pass from the dimensionless geometric parameters of the resonator to their real values. Thus, all the geometric parameters of the resonator are determined. Figure 2 shows an example of the selection of resonator parameters with a natural frequency of $500 \mathrm{~Hz}$ when the relative length of the neck is taken equal to 1 .

It can be also selected another sequence for selecting the geometric parameters of the Helmholtz resonator. For example, when we are limited in the size of the resonator, we can set its volume. It is also assumed that natural frequency of the resonator is given. Then, passing to the relative values, we can determine the relative bandwidth $\Delta \mu$ of the resonator. Then, using the nomogram in Figure 3, the remaining geometric parameters of the resonator can be determined.

\section{Conclusion}

1. The use of dimensionless parameters made it possible to develop a method for the unambiguous selection of the geometric parameters of the Helmholtz resonator in a duct with specified acoustic characteristics.

2. A simplified approach to the selection of parameters of the Helmholtz resonator with a short neck allows one to select the geometric parameters of the resonator for both linear and non-linear operating modes;

3. A general approach to the selection of Helmholtz resonator parameters on linear operating modes allows determining the geometric parameters of the Helmholtz resonator, which provide the specified (minimum possible) transmission loss at the resonator natural frequency.

\section{References}

1. Noise control in the industry, Ed., J.D. W ebb, (Sound Reserch Laboratory, UK, 1976)

2. D. V. Bazhenov, L. A. Bazhenova, A. V. Rimskiy-K orsakov, A coust. Phys. 46 (3), 306 (2000)

3. N. G. Kanev, A coust. Phys. 64 (6), 752 (2018)

4. N. G. Kanev, A coust. Phys. 66 (3), 327 (2020)

5. U. Ingard, J. A coust. Soc. A m. 25 (6), 1037 (1953)

6. D. D. Davis, G. M. Stokes, D. M oor, G. L. Stevens, NASA. Rep. 1192, 48 p (1954)

7. M. A lster, J. Sound Vib. 24 (1), 63 (1972)

8. P. K. Tang, W. A. Sirignano, J. Sound V ib. 26 (2), 247 (1973)

9. R. L. Panton, J. M. M iller, J. A coust. Soc. A m. 57 (6), 1533 (1975)

10. A. D. Lapin, A coust. Phys. 21 (3), 337 (1975)

11. R. C. Chanaud, J. Sound Vib. 178 (3), 337 (1994)

12. A. Selamet, N. S. Dickey, J. M. N ovak, J. Sound V ib. 187 (2), 358 (1995)

13. N. S. Dickey, A. Selament, J. Sound V ib. 195 (3), 512 (1996)

14. A. Selamet, P. M. Radovich, N. S. Dickey, J. M. Novak, J. A coust. Soc. A m. 101 (1), 41 (1997) 
15. R. C. Chanaud, J. Sound Vib. 204 (5), 829 (1997)

16. A. I. K omkin, RA O Y earbook, 12, 77 (2011)

17. A. K. Trambovetskaya, A. I. Bykov, A. I. K omkin, Improving the methodology for the selection of geometric parameters of Helmholtz resonator, in Proceedings of the III Conference of $Y$ oung Scientists and Specialists on Environmental A coustics, A SO2018, 18 M ay 2018, M oscow, Russia (2018) [in Russsian]

18. A. K. Trambovetskaya, A I. Bykov, A . I. Komkin, The selection of geometric parameters Helmholtz resonator, in Proceedings of the IV Conference of $Y$ oung Scientists and Specialists on Environmental A coustics, A SO-2019, 24 M ay 2019, M oscow, Russia (2019) [in Russsian]

19. A . I. Komkin, A. I. Bykov, A coust. Phys. 62 (3), 277 (2016)

20. A . I. Komkin, M. A . M ironov, A. I. Bykov, A coust. Phys. 63 (4), 356 (2017)

21. A. I. Komkin, M. A. M ironov, A coust. Phys. 61 (4), 442 (2015)

22. A. K omkin, A. Bykov, M. M ironov, Experimental study of nonlinear acoustic impedance of circular orifices, J. A coust. Soc. A m. (2020) (in print). 